\title{
Letter
}

\section{Can Higgs inflation be saved with high-scale supersymmetry?}

\author{
Sibo Zheng ${ }^{\mathrm{a}}$ (1) \\ Department of Physics, Chongqing University, Chongqing 401331, People's Republic of China
}

Received: 21 July 2015 / Accepted: 5 October 2015 / Published online: 14 October 2015

(C) The Author(s) 2015. This article is published with open access at Springerlink.com

\begin{abstract}
It is shown whether Higgs inflation can be saved as high-scale supersymmetry critically depends on the magnitude of the non-minimal coupling constant $\xi$. For small $\xi \leq 500$, the threshold correction at scale $M_{P} / \xi$ is constrained with high precision. Its magnitude is in the narrow range of $(-0.03,-0.02)$ and $(-0.05,-0.04)$ for the wino and higgsino-singlino dark matter, respectively. In the large$\xi$ region with $\xi \geq 10^{4}$, such high-scale supersymmetry is excluded by a too large threshold correction as required by Higgs inflation.
\end{abstract}

\section{Introduction}

The collider facilities such as the Large Hadron Collider (LHC) and astrophysical experiments are two main tools for exploring new physics beyond the standard model (SM). Recently, the Planck Collaboration, designed to detect the cosmic microwave background (CMB) temperature anisotropy and polarization, has reported the latest value of the tensor-to-scalar ratio $r \leq 0.11$ at $95 \% \mathrm{CL}[1,2]$. This data excludes a few well-known inflation models such as quadratic inflation, but it still allows some simple examples such as Starobinsky-like inflation [3], $\alpha$-attractor inflation [4], and Higgs inflation [5].

Among these survivors, Higgs inflation is rather special due to two considerations. First, there is only one new parameter $\xi$ in this model in the Lagrangian in this model, which reads, as in the Jordan frame,

$\mathcal{L}_{J}=\frac{M^{2}+\xi h^{2}}{2} R-\frac{1}{2}(\partial h)^{2}-\frac{\lambda_{H}}{4}\left(h^{2}-v^{2}\right)^{2}$.

Here, $\xi$ measures the non-minimal coupling between the Higgs scalar $h$ and gravity. It was found that the Higgs scalar potential $V_{E}(h)$ in the Einstein frame rapidly approaches a plateau potential in the large field region $h>M_{P} / \sqrt{\xi}$, and the model predicts that, for an e-folding number $N=60$,

a e-mail: sibozheng.zju@gmail.com $n_{s} \simeq 0.970, \quad r \simeq 0.0033$,

which is in perfect agreement with the Planck 2015 data $[1,2]$. In terms of the present cosmological data, $M$ in Eq. (1) approximates the reduced Planck mass $M_{P}=2.4 \times 10^{18}$ $\mathrm{GeV}$ for our present universe. Moreover, unlike Starobinskylike inflation or $\alpha$-attractor inflation, it is obvious that Higgs inflation is of special interest from the viewpoint of particle physics.

Unfortunately, there are two arguments against Higgs inflation. The first shows that the SM is not valid above the scale $M_{P} / \xi$ [6-8]. It implies that the plateau potential $V(h)$ at large field value, $h>M_{P} / \sqrt{\xi}$, may be significantly modified by the ultra-violet completion so that realistic inflation driven by the Higgs field cannot occur at all. It is proposed in [9] that a scale symmetry in the ultra-violet completion may be the prescription to this problem. The second argument arises because of the discovery of the Higgs mass $m_{h}=125.5 \pm 0.5 \mathrm{GeV}$ [10-12], which implies that the SM Higgs quartic coupling $\lambda_{H}$ at the weak scale is not large enough, so that $\lambda_{H}<0$ above the high-energy scale of $10^{9}-10^{11} \mathrm{GeV}$. The uncertainty about this critical scale mainly arises from the uncertainty of the top quark mass, as the renormalization group equation (RGE) for $\lambda_{H}$ is very sensitive to the top Yukawa coupling. Obviously, a positive $\lambda_{H}$ is required during the inflationary epoch. This problem can be solved through introducing some new fields into the $\mathrm{SM}$, which give rise to either a correct threshold correction to $\lambda_{H}$ or slowing down the RG evaluation for $\lambda_{H}$ along the high energy scale; see, e.g., [13,14].

In this letter, we consider saving the Higgs inflation with high-scale supersymmetry (SUSY). High-scale SUSY can provide the observed Higgs mass at the LHC and a stable DM candidate [15]. However, the detection of such models at colliders is unpromising. In this sense the astrophysical probe is an important direction. Fortunately, they can be probed directly or indirectly in the light of the cosmological observations on the early universe $[16,17]$. 
We identify the cut-off scale $M_{P} / \xi$ as the typical mass scale $\tilde{m}$ in the SUSY mass spectrum. Above the scale $\tilde{m}=$ $M_{P} / \xi$ supergravity is the natural ultra-violet (UV) completion, and as regards the matter content the same as the nextto-minimal SUSY model (NMSSM); it may maintain the plateau potential [18]. For earlier discussions, see [19-21]. On the other hand, the SUSY dark matter (DM), whose mass is around the weak scale, is the natural choice on the new fields added to the SM below the cut-off scale $\tilde{m}$. In this paper, we consider two possibilities [15], where the wino $\tilde{w}$ and the higgsino-singlino mixing state serves as the DM, respectively.

The paper is organized as follows. In the next section, we discuss the RGE for $\lambda_{H}$ in two classes of high-scale SUSY below the scale $\tilde{m}$. In Sect. 3, we discuss embedding Higgs inflation into supergravity, where we uncover the constraint on the parameters $\lambda_{H}$ and $\xi$ at the end of inflation $h_{\text {end }}=$ $M_{P} / \sqrt{\xi}$, arising from the present cosmological data. In Sect. 4 , we discuss the constraint on the SUSY mass spectrum in terms of the constraint on the threshold correction to $\lambda_{H}$. Finally, we discuss our results in Sect. 5.

\section{RGE for $\lambda_{H}$ below scale $M_{P} / \xi$}

Below the scale $\tilde{m}$, the effective theory is described by the SM together with either wino-like or higgsino-singlino-like DM. In this section we use the 2-loop RGEs for relevant couplings for our analysis. We refer the reader to the appendix in our previous work [15], where the 2-loop RGEs for SM gauge and Yukawa couplings, and Higgs quartic coupling in models of $\mathrm{SM}+\tilde{w}$ and $\mathrm{SM}+\tilde{h} / \tilde{s}$ are explicitly shown. We ignore other SM Yukawa couplings in the following discussion. For the calculation of beta functions, see the references therein.

Figure 1 shows the RG running for $\lambda_{H}$ in high-scale SUSY discussed in this letter, which is modified by the SUSY DM (wino or higgisno-singlino mixing state) with the mass of the weak scale being compared with the SM. In this figure, black, blue, and green curves corresponds to the $\mathrm{SM}, \mathrm{SM}+\tilde{w}$, and $\mathrm{SM}+\tilde{h} / \tilde{s}$, respectively. The solid lines refer to the central value $m_{t}=173.1 \mathrm{GeV}, m_{h}=125.5 \mathrm{GeV}$, and $\alpha_{s}\left(m_{Z}\right)=$ 0.1184 , while the dotted lines show the uncertainty due to the top quark mass with $1 \sigma$ deviation. From Fig. 1 we observe that a more positive $\lambda_{H}$ is obtained in high-scale SUSY with wino DM for a smaller top quark pole mass $m_{t}=172.2 \mathrm{GeV}$ along the RG running to the scale $M_{P} / \xi$. This is mainly due to the correction to the SM beta function $\beta_{\lambda_{H}}$ induced by the wino DM.

Unlike the case with the wino DM, the sign of the correction induced by the higgsino-singlino DM is only positive when the model parameter $g_{\lambda}$ is small. Otherwise, for large $g_{\lambda} \geq 0.4$ the sign of the correction will reverse and $\lambda_{H}$ will become negative more rapidly along the RG running to scale

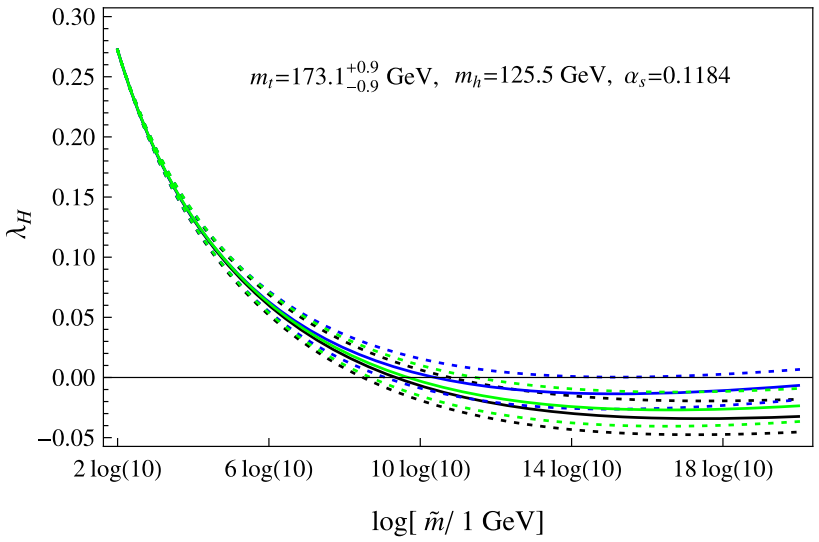

Fig. 1 Two-loop RG running for $\lambda_{H}$ in the SM (black), SM+ $\tilde{w}$ (blue) and $\mathrm{SM}+\tilde{h} / \tilde{s}$ (green), respectively. The solid lines correspond to the central value $m_{t}=173.1 \mathrm{GeV}, m_{h}=125.5 \mathrm{GeV}$ and $\alpha_{s}\left(m_{Z}\right)=$ 0.1184 . The dotted lines show the uncertainty due to the top quark mass with $1 \sigma$ deviation. In the model of $\mathrm{SM}+\tilde{h} / \tilde{s}$, we have chosen a small value $g_{\lambda}\left(m_{Z}\right)=0.2$. See the text for the reason for this choice

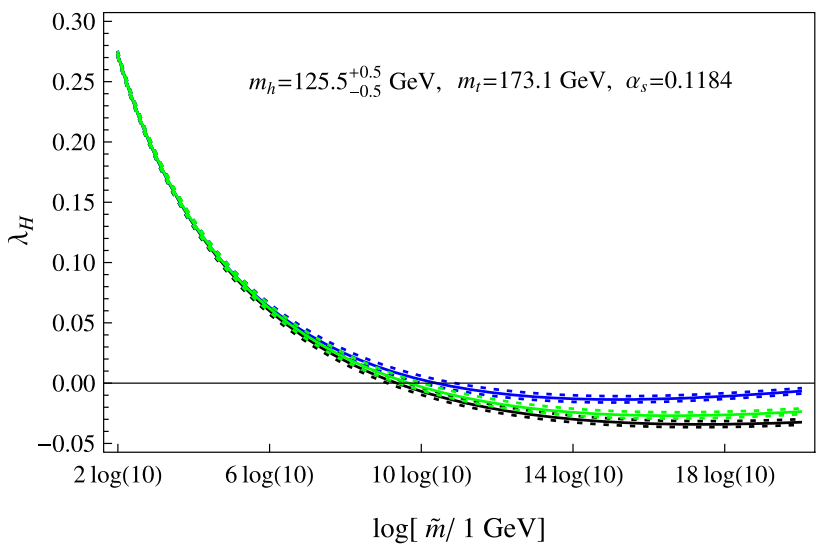

Fig. 2 Same as Fig. 1 but with $m_{t}=173.1 \mathrm{GeV}$ and $m_{h}=125.5 \pm 0.5$ $\mathrm{GeV}$

$M_{P} / \xi$. This implies that the correction in this case is upper bounded.

Figure 2 shows the sensitivity of the RG running for $\lambda_{H}$ to the Higgs mass. One finds that for about $\sim 0.5 \mathrm{GeV}$ deviation to the central value $m_{h}=125.5 \mathrm{GeV}$ the correction in each case is tiny. Among the three models, $\lambda_{H}$ approaches zero mostly in the case with wino DM, similarly to what Fig. 1 indicates.

In summary, for about $1 \sigma$ deviation to the central value of top quark mass, $\lambda_{H}$ is tuned to be more positive in high-scale SUSY with the low-energy theory below the scale $M_{P} / \xi$ described by either $\mathrm{SM}+\tilde{w}$ or $\mathrm{SM}+$ higgsino-singlino $\mathrm{DM}$ being compared with the SM. As we will see in the next section, a more positive $\lambda_{H}$ at scale $M_{P} / \xi$ is favored by Higgs inflation. This is so because the threshold correction at the scale $M_{P} / \xi$ may be unable to tune $\lambda_{H}$ into a positive parameter above this scale as required by Higgs inflation. 


\section{Embedding Higgs inflation into supergravity}

We proceed to a discussion of the constraint arising from the condition of a plateau potential for the Higgs scalar in the context of supergravity, which is automatically the ultra-violet completion. For this purpose, we focus on the scalar-gravity part of the supergravity Lagrangian, which is defined by the frame function $\Omega\left(z_{i}, \bar{z}_{i}\right)$, the Kähler potential $K\left(z_{i}, \bar{z}_{i}\right)$ and the superpotential $W\left(z_{i}\right)$. We use $z_{i}$ to label the chiral superfields including the two Higgs doublets $H_{u, d}$.

We follow the notation and conventions in [18], where the Kähler potential and frame function are given in units of the Planck mass by

$$
\begin{aligned}
& K\left(z_{i}, \bar{z}_{i}\right)=-3 \log (\Omega), \\
& \Omega\left(z_{i}, \bar{z}_{i}\right)=1-\frac{1}{3} \delta_{i j} z^{i} z^{\bar{j}}+\cdots
\end{aligned}
$$

Given the explicit form of these functions, the scalar potential in the Einstein frame is directly derived from the well-known formula ${ }^{1}$

$V_{E}=\mathrm{e}^{K}\left(D_{i} W K^{i \bar{j}} D_{\bar{j}} \bar{W}-3 W \bar{W}\right)+V_{E}^{(D)}$,

where $D_{i} W=\partial_{i} W+K_{i} W, V_{E}^{(D)}$ represents the D-term contribution.

The first attempt to embed Higgs inflation into supergravity was shown in [20]. In the light of [20] there are two important observations. (1) The supergravity version of Eq. (1) requires a holomorphic function $X=-\frac{1}{2} \chi H_{u} H_{d}+$ h.c in order to reproduce the $\xi$-term. $\chi$ is a dimensionless coupling constant. (2) The matter content of MSSM is not viable for the Higgs inflation. By following this line, the authors in [18] proposed that the matter context of NMSSM is a realistic choice, in which the potential in the NMSSM depends on three complex superfields,

$$
\begin{aligned}
z_{i} & =\left\{S, H_{u}^{0}, H_{d}^{0}\right\} \\
& =\left\{s \mathrm{e}^{i \alpha} / \sqrt{2}, h \cos \beta \mathrm{e}^{i \alpha_{1}} / \sqrt{2}, h \sin \beta \mathrm{e}^{i \alpha_{2}} / \sqrt{2}\right\},
\end{aligned}
$$

as long as the scalar $s$ in the singlet superfield $S$ can be stabilized at $s=0$ and the $D$-flat condition $\beta=\pi / 4$ is satisfied. This idea was first considered in [20], but a new $\zeta$ term should be added to the frame function [18] for stabilizing the $s$ scalar.

In summary, the frame function and superpotential for the purpose of Higgs inflation are given by, respectively,

${ }^{1}$ Correspondingly, the scalar potential in the Jordan frame is given by $V_{J}=\Omega^{2} V_{E}$.

$$
\begin{aligned}
\Omega\left(z_{i}, \bar{z}_{i}\right)= & 1-\frac{1}{3}\left(\left|H_{u}^{0}\right|^{2}+\left|H_{d}^{0}\right|^{2}+|S|^{2}\right) \\
& +\frac{\zeta}{3}|S|^{4}-\left(\frac{1}{2} \chi H_{u}^{0} H_{d}^{0}+\text { h.c }\right), \\
W\left(z_{i}, \bar{z}_{i}\right)= & \lambda S H_{u}^{0} H_{d}^{0}+\frac{\rho}{3} S^{3} .
\end{aligned}
$$

Substituting Eq. (6) into Eq. (4) gives rise to the potential

$V_{E}=V_{E}^{(F)}=\frac{9 \lambda^{2} h^{4}}{\left(3 \chi h^{2}-2 h^{2}+6\right)^{2}}$,

for $s=0$ and $\beta=\pi / 4$. Here we have used $\left.W\right|_{s=0}=0$. For more details as regards the stabilization of $s$ and angles in Eq. (5), see $[18,21]$. In the region $\chi h^{2}>>1>>h^{2}$, Eq. (7) approaches $(\lambda / \chi)^{2}$ (in units of the Planck mass), which verifies our statements above. Moreover, in terms of Eq. (7) one can also verify Eq. (2).

The parameters $\xi$ and $\lambda_{H}$ in Eq. (1) are related to the parameters $\chi$ and $\lambda$ in Eq. (6) as

$\xi=-\frac{1}{6}+\frac{1}{4} \chi, \quad \lambda_{H}\left(\mu_{I}\right)=\frac{\lambda^{2}}{4}$.

Here $\mu_{I}$ denotes the RG scale corresponding to inflation. They are constrained by the present cosmological data as follows. Recall that $V_{E}^{1 / 4}=\left(24 \pi^{2} M_{P}^{4} \epsilon A_{S}\right)^{1 / 4}$, where $A_{s}$ is the amplitude of the power spectrum of the curvature perturbation and $\epsilon=r / 16$ in the context of single field inflation. For $A_{S}^{1 / 2} \simeq 3.089 \times 10^{-5}$ as reported by Plank Collaboration [22] one obtains

$\xi \simeq 58789 \sqrt{\lambda_{H}\left(\mu_{I}\right)}$.

\section{Constraints on SUSY mass spectrum}

Until now, we have obtained the value of $\lambda_{H}$ below the RG scale $\mu=M_{P} / \xi$ (as shown in Figs. 1 and 2) and above the RG scale $M_{P} / \sqrt{\xi}$ (as shown in Eq. (9)) given a choice on $\xi$. The effective theory at the intermediate scale between scale $M_{P} / \xi$ and $M_{P} / \sqrt{\xi}$ is described by the SM together with gauginos and squarks. Other SUSY particles such as higgsinos and charged Higgs particles have masses of order $M_{P} / \sqrt{\xi}[18,21]$, so they should be integrated out at this intermediate energy scale [25], especially for the discussion of the RGE for $\lambda_{H}$.

The threshold correction $\delta \lambda_{H}$ at scale $\tilde{m}=M_{P} / \xi$ arising from integrating out gauginos and squarks can be determined in terms of the differences between the values of $\lambda_{H}$ below and above the scale $\tilde{m}=M_{P} / \xi$,

$\lambda_{H}(\tilde{m}-\epsilon)=\lambda_{H}(\tilde{m}+\epsilon)+\delta \lambda_{H}\left(m_{g_{1}}, m_{g_{3}}, m_{\tilde{q}_{i}}\right)$,

where $0<\epsilon<<1$, and $m_{g_{1}}, m_{g_{3}}$, and $m_{\tilde{q}_{i}}$ refer to the gluino mass, bino mass, and squark mass, respectively. As we will see below, the threshold correction $\delta \lambda_{H}\left(m_{g_{1}}, m_{g_{3}}, m_{\tilde{q}_{i}}\right)$ can 
be measured at high precision, so it is a new and useful factor to constrain the GUT-scale SUSY mass spectrum.

The value of $\lambda_{H}\left(M_{P} / \xi+\epsilon\right)$ in Eq. (10) is determined by the RGE for $\beta_{\lambda_{H}}$ in the effective theory at the intermediate scale, with the boundary value at the end of inflation

$\lambda_{H}\left(M_{P} / \sqrt{\xi}\right)=\lambda_{H}\left(\mu_{I}\right)+\delta \lambda_{H}\left(m_{\tilde{h}_{u, d}}, m_{s}\right)$.

Here, we have ignored the effects due to higgsino-induced operators with mass dimension higher than four, which are at least one order of magnitude smaller than the threshold correction in our case. ${ }^{2}$

The threshold correction $\delta \lambda_{H}\left(m_{\tilde{h}_{u, d}}, m_{S}\right)$ in Eq. (11) arises from the heavy higgsinos and singlet $s$, which are given by, respectively [24],

$$
\begin{aligned}
\left.\delta \lambda_{H}\right|_{\tilde{h}_{u, d}} & \simeq-\frac{1}{6} \cos ^{2}(2 \beta)\left(\frac{9}{25} g_{1}^{4}+g_{2}^{4}\right) \ln \left(\frac{\mu^{2}}{\tilde{m}^{2}}\right), \\
\left.\delta \lambda_{H}\right|_{s} & \simeq 2 \lambda_{H}\left(\mu_{I}\right) \sin ^{2}(2 \beta),
\end{aligned}
$$

where we have assumed that the A-term $A_{s}$ is smaller than $m_{s}$. Substituting the stabilized value $\beta=\pi / 4$ into Eq. (12) we find that $\lambda_{H}\left(M_{P} / \sqrt{\xi}\right) \simeq \frac{5}{2} \lambda_{H}\left(\mu_{I}\right)$. As long as the mass scale $m_{\beta}$, which is of order $M_{P} / \sqrt{\xi}$, is far larger than the Hubble parameter $H$ and the reheating temperature after inflation [23], $\beta$ rapidly approaches the stabilized value during and after inflation.

The matter content of the effective theory at the intermediate scale is composed of SM fermions, squarks, gauginos, and electrically neutral scalars in the Higgs sector. The beta function coefficients $b_{i}$ for the SM gauge coupling in this effective theory are given by, respectively,

$b_{1}=\frac{n}{2}, \quad b_{2}=-\frac{3}{2}+\frac{n}{2}, \quad b_{3}=-\frac{9}{4}+\frac{n}{2}$,

where $n=3$ is the number of SM fermion generations. In terms of Eq. (13) and one-loop RGEs for $\lambda_{H}$ and SM top Yukawa coupling we show in Fig. 3 the constraint on the magnitude of $\delta \lambda_{H}\left(m_{g_{1}}, m_{g_{3}}, m_{\tilde{q}_{i}}\right)$ for the range $300 \leq \xi \leq$ 50000. It is shown that the value for $\delta \lambda_{H}\left(m_{g_{1}}, m_{g_{3}}, m_{\tilde{q}_{i}}\right)$ as required by Higgs inflation is very sensitive to the parameter $\xi$.

In the light of Fig. 3 we conclude that for small nonminimal coupling, $\xi \leq 500$, the threshold correction at scale

\footnotetext{
$\overline{2}$ Operators of type $c_{H G}|H|^{2} F_{\mu \nu} F^{\mu \nu} / M^{2}$ with mass dimension six, as induced by heavy SUSY particles with mass $M$, contribute to the leading corrections to the RGEs for SM EW gauge coupling [26,27]. As a result, they modify the RGE for $\lambda_{H}$ indirectly, the significance of which is determined by the ratio $\left(m_{h}^{2}\right)_{\text {eff }} / M^{2}$, as seen from the modified beta function with SM gauge coupling, $16 \pi^{2} \delta \beta_{g_{i}} \sim c_{H G} g_{i}\left(m_{h}^{2}\right)_{\mathrm{eff}} / M^{2}$. For the three stages we set in this paper, the modification to the RGE for $\lambda_{H}$ during inflation is maximal, with the heavy SUSY particle identified as $\tilde{h}_{\mu, d}$. In contrast, the modification after inflation is only mild. In our case, $\left(m_{h}^{2}\right)_{\text {eff }} \leq H$ and $M \sim M_{P}$, which implies that this modification is smaller than the threshold correction $\delta \lambda_{H}\left(m_{\tilde{h}_{u, d}}, m_{S}\right)$.
}

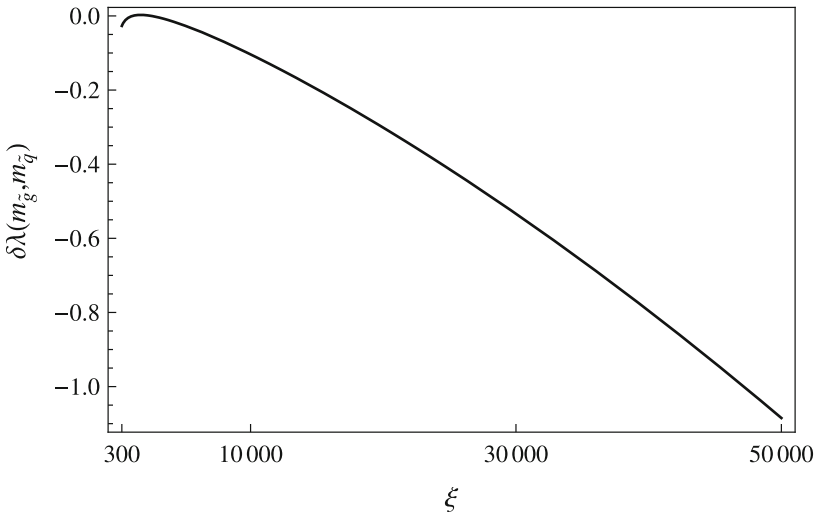

Fig. 3 Threshold correction $\delta \lambda_{H}\left(m_{g_{1}}, m_{g_{3}}, m_{\tilde{q}_{i}}\right)$ in the wino DM model. We have chosen $m_{t}=172.2 \mathrm{GeV}, m_{h}=125.5 \mathrm{GeV}$, and $\alpha_{s}\left(m_{Z}\right)=0.1184$. A similar plot can be obtained for the case with higgsino-singlino DM

$M_{P} / \xi$ induced by Higgs inflation is about $-0.03 \sim-0.02$ and $-0.05 \sim-0.04$ in wino and higgsino-singlino DM, respectively. The uncertainties mainly arise from the uncertainty of the top quark pole mass. In the large $\xi$ region with $\xi \geq 1 \times 10^{4}$, the required threshold correction is $\leq-0.1$, which is too large to exclude such models.

\section{Discussion and summary}

So far we have only discussed the constraint on the threshold correction $\delta \lambda$ at scale $M_{P} / \xi$ in the case for wino DM. In the case for higgsino-singlino DM the one-loop RGEs for relevant couplings at the intermediate scale are the same as in Sect. 4. So the conclusion there is also applied to the model of higgsino-singlino DM. Actually, given the same $\xi$ it is expected that the threshold correction for the case with higgsino-singlino DM is larger than what is shown in Fig. 3.

One may wonder whether adopting larger $m_{t}$ than 172.2 $\mathrm{GeV}$ can reduce the threshold correction in Fig. 3. Figures 1 and 2 show that $\lambda_{H}\left(M_{P}-\epsilon\right)$ becomes more negative when one chooses larger $m_{t}$, and the value of $\lambda\left(M_{P} / \xi+\epsilon\right)$, which is positive, will be smaller simultaneously. Therefore the deviation to the threshold correction is not too obvious to violate our conclusions.

In summary, the answer to the question whether Higgs inflation can be saved by high-scale SUSY critically depends on the magnitude of $\xi$. For small non-minimal coupling $\xi \leq 500$, the threshold correction at scale $M_{P} / \xi$ required by Higgs inflation is constrained to high precision, the magnitude of which is about $-0.03 \sim-0.02$ and $-0.05 \sim-0.04$ for the wino and higgsino-singlino DM, respectively. The uncertainties mainly arise from the uncertainty of the top quark pole mass. This amount of threshold correction can be explained, for example, for universal squark masses $m_{\tilde{q}} \simeq$ 
$\frac{M_{P}}{6 \xi}$ with vanishing $A_{t}$ terms. For large $\xi \sim 10^{4}$, the required threshold correction is smaller than $\simeq-0.1$, which excludes such high-scale SUSY. Finally, we believe our analysis can be applied to Split SUSY, although this model is more complicated in comparison with what have been discussed here.

Acknowledgments We would like to thank the referee for valuable suggestions. The work is supported in part by National Natural Science Foundation of China under grant No. 11247031 and 11405015.

Open Access This article is distributed under the terms of the Creative Commons Attribution 4.0 International License (http://creativecomm ons.org/licenses/by/4.0/), which permits unrestricted use, distribution, and reproduction in any medium, provided you give appropriate credit to the original author(s) and the source, provide a link to the Creative Commons license, and indicate if changes were made.

Funded by SCOAP ${ }^{3}$.

\section{References}

1. P.A.R. Ade et al., [Planck Collaboration], Planck 2015 results. XIII. Cosmological parameters. arXiv:1502.01589 [astro-ph.CO]

2. P.A.R. Ade et al., [Planck Collaboration], Planck 2015 results. XX. Constraints on inflation. arXiv:1502.02114 [astro-ph.CO]

3. A.A. Starobinsky, A new type of isotropic cosmological models without singularity. Phys. Lett. B 91, 99 (1980)

4. R. Kallosh, A. Linde, Planck, LHC, and $\alpha$-attractors. arXiv:1502.07733 [astro-ph.CO]

5. F.L. Bezrukov, M. Shaposhnikov, The standard model Higgs boson as the inflaton. Phys. Lett. B 659, 703 (2008). arXiv:0710.3755 [hep-th]

6. C.P. Burgess, H.M. Lee, M. Trott, Power-counting and the validity of the classical approximation during inflation. JHEP 0909, 103 (2009). arXiv:0902.4465 [hep-ph]

7. J.L.F. Barbon, J.R. Espinosa, On the naturalness of Higgs inflation. Phys. Rev. D 79, 081302 (2009). arXiv:0903.0355 [hep-ph]

8. C.P. Burgess, H.M. Lee, M. Trott, Comment on Higgs inflation and naturalness. JHEP 1007, 007 (2010). arXiv:1002.2730 [hep-ph]

9. F. Bezrukov, A. Magnin, M. Shaposhnikov, S. Sibiryakov, Higgs inflation: consistency and generalisations. JHEP 1101, 016 (2011). arXiv:1008.5157 [hep-ph]

10. S. Chatrchyan et al., [CMS Collaboration], Measurement of the properties of a Higgs boson in the four-lepton final state. Phys. Rev. D 89(9), 092007 (2014). arXiv:1312.5353 [hep-ex]
11. V. Khachatryan et al., [CMS Collaboration], Observation of the diphoton decay of the Higgs boson and measurement of its properties. Eur. Phys. J. C 74(10), 3076 (2014). arXiv: 1407.0558 [hep-ex]

12. G. Aad et al., [ATLAS Collaboration], Measurement of the Higgs boson mass from the $H \rightarrow \gamma \gamma$ collision data. Phys. Rev. D 90(5), 052004 (2014). arXiv:1406.3827 [hep-ex]

13. J. Elias-Miro et al., Stabilization of the electroweak vacuum by a scalar threshold effect. JHEP 1206, 031 (2012). arXiv:1203.0237 [hep-ph]

14. H.J. He, Z.Z. Xianyu, Extending Higgs inflation with TeV scale new physics. JCAP 1410, 019 (2014). arXiv:1405.7331 [hep-ph]

15. S.Zheng, On dark matter selected high-scale supersymmetry. JHEP 1503, 062 (2015). arXiv:1409.2939 [hep-ph]

16. D. Baumann, D. Green, Signatures of supersymmetry from the Early Universe. Phys. Rev. D 85, 103520 (2012). arXiv:1109.0292 [hep-th]

17. S. Zheng, The Early Universe with High-Scale Supersymmetry. arXiv:1409.7462 [hep-ph]

18. S. Ferrara et al., Superconformal symmetry, NMSSM, and inflation. Phys. Rev. D 83, 025008 (2011). arXiv:1008.2942 [hep-th]

19. I. Ben-Dayan, M.B. Einhorn, Supergravity Higgs inflation and shift symmetry in electroweak theory. JCAP 1012, 002 (2010). arXiv: 1009.2276 [hep-ph]

20. M.B. Einhorn, D.R.T. Jones, Inflation with non-minimal gravitational couplings in supergravity. JHEP 1003, 026 (2010). arXiv:0912.2718 [hep-ph]

21. S. Ferrara et al., Jordan frame supergravity and inflation in NMSSM. Phys. Rev. D 82, 045003 (2010). arXiv:1004.0712 [hepth]

22. P.A.R. Ade et al., [Planck Collaboration], Planck 2013 results. XVI. Cosmological parameters. Astron. Astrophys. 571, A16 (2014). arXiv:1303.5076 [astro-ph.CO]

23. J. Garcia-Bellido, D.G. Figueroa, J. Rubio, Preheating in the standard model with the Higgs-inflaton coupled to gravity. Phys. Rev. D 79, 063531 (2009). arXiv:0812.4624 [hep-ph]

24. G.F. Giudice, A. Strumia, Probing high-scale and split supersymmetry with Higgs mass measurements. Nucl. Phys. B 858, 63 (2012). arXiv:1108.6077 [hep-ph]

25. A. De Simone, M.P. Hertzberg, F. Wilczek, Running inflation in the standard model. Phys. Lett. B 678, 1 (2009). arXiv:0812.4946 [hep-ph]

26. C.P. Burgess, S.P. Patil, M. Trott, On the predictiveness of singlefield inflationary models. JHEP 1406, 010 (2014). arXiv: 1402.1476 [hep-ph]

27. E.E. Jenkins, A.V. Manohar, M. Trott, Renormalization group evolution of the standard model dimension six operators I: formalism and lambda dependence. JHEP 1310, 087 (2013). arXiv:1308.2627 [hep-ph] 